\title{
FUSE: Multi-Faceted Set Expansion by Coherent Clustering of Skip-grams ${ }^{\star}$
}

\author{
Wanzheng Zhu ${ }^{1}[\varangle]$, Hongyu Gong ${ }^{1}$, Jiaming Shen ${ }^{1}$, Chao Zhang ${ }^{2}$, Jingbo \\ Shang ${ }^{3}$, Suma Bhat ${ }^{1}$, and Jiawei Han ${ }^{1}$ \\ 1 University of Illinois at Urbana-Champaign, IL, USA \\ \{wz6, hgong6, js2, spbhat2, hanj\}@illinois.edu \\ 2 Georgia Institute of Technology, GA, USA \\ chaozhang@gatech.edu \\ 3 University of California San Diego, CA, USA \\ jshang@ucsd.edu
}

\begin{abstract}
Set expansion aims to expand a small set of seed entities into a complete set of relevant entities. Most existing approaches assume the input seed set is unambiguous and completely ignore the multi-faceted semantics of seed entities. As a result, given the seed set \{ Canon", "Sony", "Nikon"\}, previous models return one mixed set of entities that are either Camera Brands or Japanese Companies. In this paper, we study the task of multi-faceted set expansion, which aims to capture all semantic facets in the seed set and return multiple sets of entities, one for each semantic facet. We propose an unsupervised framework, FUSE, which consists of three major components: (1) facet discovery module: identifies all semantic facets of each seed entity by extracting and clustering its skip-grams, and (2) facet fusion module: discovers shared semantic facets of the entire seed set by an optimization formulation, and (3) entity expansion module: expands each semantic facet by utilizing a masked language model with pre-trained BERT models. Extensive experiments demonstrate that FUSE can accurately identify multiple semantic facets of the seed set and generate quality entities for each facet.
\end{abstract}

Keywords: Set Expansion - Multi-facetedness · Word Sense Disambiguation.

\section{Introduction}

The task of set expansion is to expand a small set of seed entities into a more complete set of relevant entities. For example, to explore all Universities in the U.S., one can feed a seed set (e.g., \{ "Stanford", "UCB", "Harvard" $\})$ to a set expansion system and then expect outputs such as "Princeton", "MIT" and "UW". Those expanded entities can benefit numerous entity-aware applications, including query suggestion [1, taxonomy construction [32, recommendation [38, and information extraction 12 23 36 37. Besides, the set expansion algorithm itself becomes a basic building block of many NLP-based systems [15[27.

* Accepted at ECML-PKDD 2020 
Previous studies on set expansion focus on returning one single set of most relevant entities. Methods have been developed to incrementally and iteratively add the entities of high confidence scores into the set. A variety of features are extracted, including word co-occurrence statistics [18, unary patterns [26, or coordinational patterns [24, from different data sources such as query log [31], web table 33 , and raw text corpus 15/26. However, all these methods assume the given seed set is unambiguous and completely ignore the multi-faceted semantics of seed entities. As a result, given a seed set \{ "apollo", "artemis", "poseidon"\} which has two semantic facets - Major Gods in Greek Mythology and NASA Missions, previous methods can only generate one mixed set of entities from these two facets, which inevitably hampers their applicabilities.

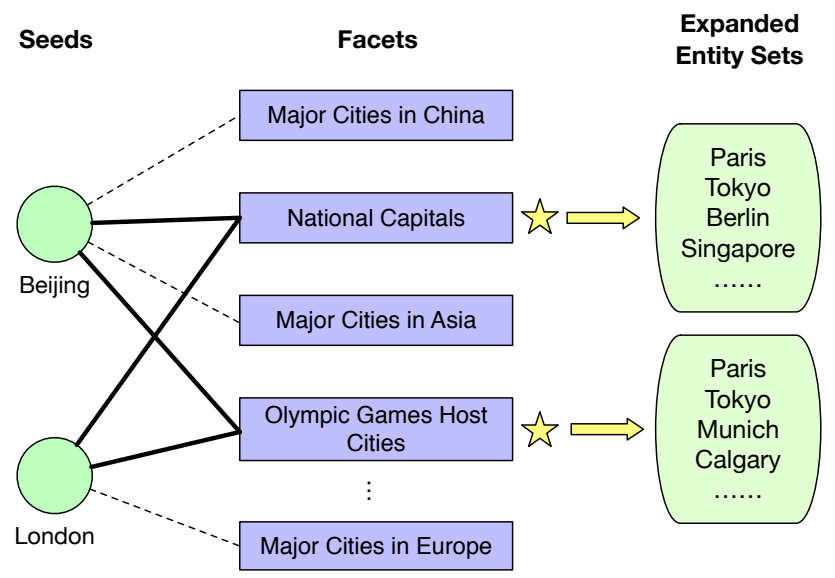

Fig. 1: An illustrative example of a multi-faceted seed set \{"Beijing", "London"\}. Facets (e.g. Major Cities in China) that do not appear in both seed entities should be eliminated in the set expansion process. As a result, we expect to output two separate entity sets: one for semantic facet National Capitals and the other one for semantic facet Olympic Games Host Cities.

In this paper, we approach the set expansion task from a new angle. Our study focuses on multi-faceted set expansion which aims to identify semantic facets shared by all seed entities and return multiple expanded sets, one for each semantic facet. The key challenge lies in the discovery of shared semantic facets from a seed set. However, the only initial attempt towards multi-facetedness, EgoSet [22], not only requires user-created ontologies as external knowledge, but also has no guarantee that their generated semantic facets are relevant to all seed entities. As an illustrative example in Fig. 11. EgoSet generates more than five facets, but only two of them are relevant to both seeds.

To handle the key challenge of multi-faceted set expansion, we propose a novel framework, FUSE, as illustrated in Fig. 2. First, we discover all possible facets 


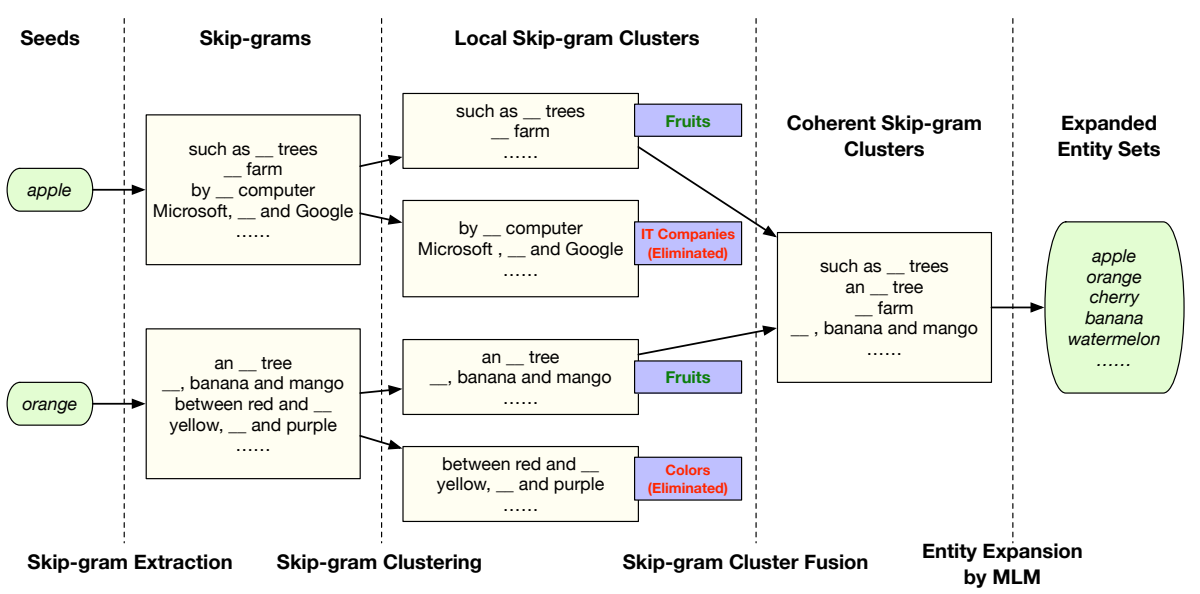

Fig. 2: Overview of FUSE. The key novelty is to discover coherent skip-gram clusters, whereas previous methods skip this stage and directly combine all skipgrams into the same pool for entity expansion.

of each seed by extracting and clustering its skip-grams. Second, we leverage an optimization formulation to discover the shared semantic facets across all seeds as coherent semantic facets. This helps eliminate those facets relevant only to a partial set of seeds. Third, based on the coherent skip-gram clusters, we utilize a masked language model (MLM) with pre-trained BERT models to generate quality entities for each semantic facet.

It is considerably complicated to evaluate such multi-faceted set expansion task, mainly because we have no prior knowledge about the number of facets in a seed set. Therefore, we are likely to observe different number of facets between the generated result and the ground truth (e.g., the ground truth may have 3 facets, while the generated result has 4 facets.). Previously proposed metric Mean-MAP (MMAP) in 22] only measures how many entities and facets in the ground truth are covered by the generated result. However, it fails to measure how noisy those generated facets are and thus it biases toward methods that output as many facets as possible. To overcome the intrinsic limitation of MMAP, we propose a more comprehensive evaluation metric, Best-Matching Average Precision (BMAP), that can not only capture the purity of generated facets but also their coverage of ground truth facets.

Our contributions are highlighted as follows.

- We identify the key challenge of multi-faceted set expansion and develop FUSE to address it 4

- We propose to determine semantic facets by clustering skip-gram contexts, and utilize an optimization formulation to discover coherent semantic facets.

\footnotetext{
${ }^{4}$ The code is available at https://github.com/WanzhengZhu/FUSE.
} 
- We propose a novel evaluation metric for multi-faceted set expansion problem, which is shown to be a more comprehensive measure.

- Extensive experiments demonstrate that our proposed framework outperforms state-of-the-art set expansion algorithms significantly.

\section{Problem Formulation}

A facet refers to one semantic aspect or sense of seed words. For example, Fruit and Technology Companies are two facets of the word "apple". Previous works study mostly single-faceted set expansion and ignore the seeds' multi-facetedness nature. In this work, we explore a better coverage of all coherent semantic facets of a seed set and study corpus-based multi-faceted set expansion.

More formally, given a seed set query $q=\left\{s_{1}, s_{2}, \ldots, s_{m}\right\}$ where $s_{i}$ is a seed and a raw text corpus $D$, our set expansion system is to find all lists of entities $\mathbb{E}=\left\{E^{(i)}, E^{(j)}, E^{(k)}, \ldots\right\}$, where $E^{(i)}=\left\{x_{1}^{(i)}, \ldots, x_{n}^{(i)}\right\}$ is relevant to the $i$-th facet $f_{i}$ of query $q$, and $x_{l}^{(i)}$ denotes an expanded entity.

\section{Model}

Our proposed FUSE framework consists of three main steps: 1) extracting and clustering skip-grams for each seed (c.f. Sec 3.1);2) discovering coherent semantic facets of a seed set (c.f. Sec 3.2); and 3) expanding entities for each semantic facet (c.f. Sec 3.3). An overview of our approach is shown in Fig. 2 and the algorithm is shown in Algorithm 1

\subsection{Skip-gram Features Extraction and Clustering}

We preprocess the raw corpus and extract skip-gram features of seed words as [26] and 22] do. Here skip-gram features are a sequence of words surrounding the seed word. Based on the distributional hypothesis [16, the semantics of a word is reflected by its neighboring skip-grams. We can derive different facets of a seed word by separating its skip-grams into different semantic clusters.

Embedding is commonly used in NLP applications to represent rich semantic information of words and phrases. We obtain the embedding for each skip-gram by simply averaging the embedding of its component words. The derivation of skip-gram embedding is another interesting research question, but it is not our focus in this work.

Now we cluster these skip-gram embeddings to discover different semantic facets of a seed word. Most clustering algorithms require the number of clusters as input, which deviates from our problem setting. Also, we note that the embedding usually lies in a high-dimension space (typically of dimension 100300 ), which leads to the poor and unstable performance of most existing nonparametric clustering algorithms 29] (e.g., MeanShift [4]). 


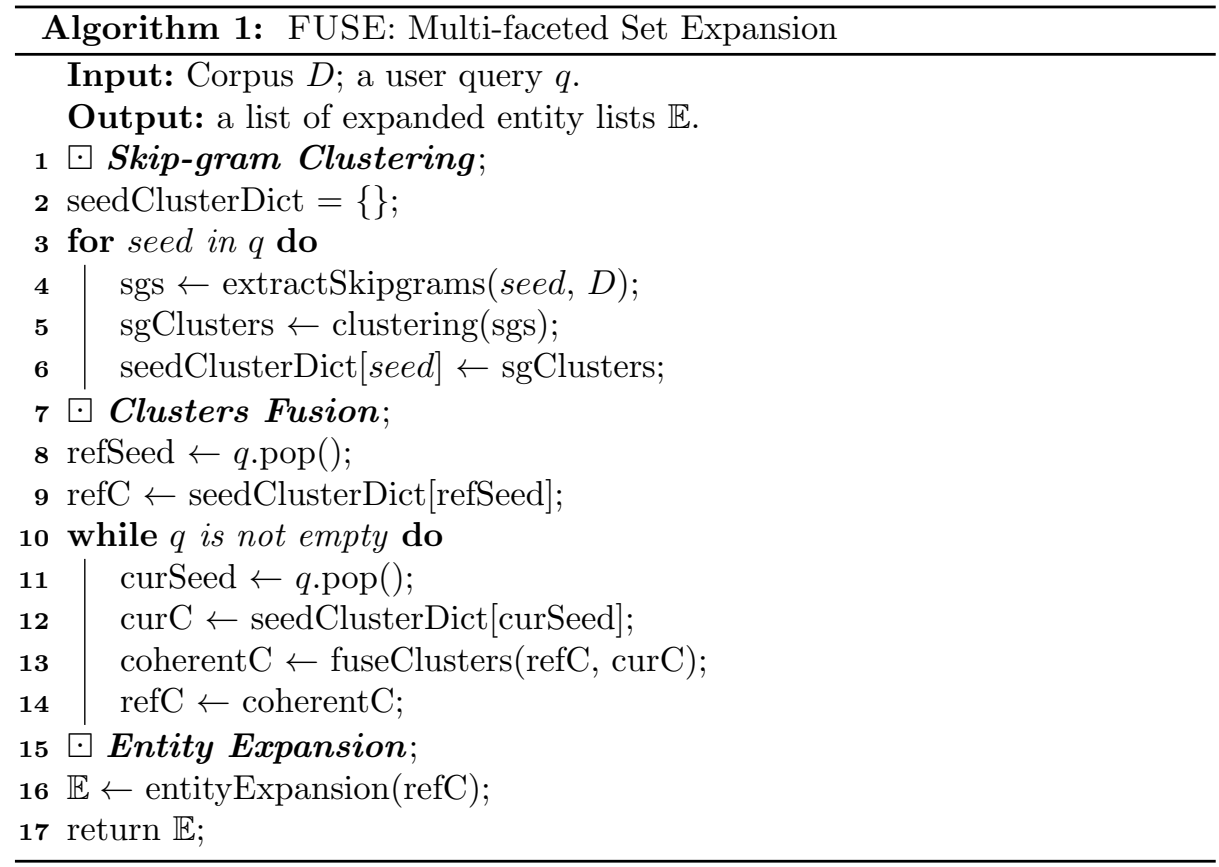

To solve the two main issues of instability and hard coded cluster numbers as mentioned above, we propose to tackle the high-dimensional embedding clustering problem by the affinity propagation algorithm [6. Specifically, we construct a complete weighted graph where each node represents a skip-gram, and the edge weight between each pair of nodes indicates the cosine similarity of their corresponding skip-gram embeddings. After the weighted graph of skip-grams is constructed, the affinity propagation algorithm [6] is applied to find the best skip-gram clusters ${ }^{5}$

Empirical results demonstrate that the affinity propagation based skip-gram clustering is able to identify a reasonable number of semantic facets (c.f. Section 4.4. We think one possible reason is that affinity propagation takes a similarity graph as its input, while most other non-parametric clustering algorithms (e.g., MeanShift [4) take skip-gram embeddings as its input. In such a clustering task, we are only interested in semantic similarities between skip-grams and do not care about the complete information of the skip-grams (e.g., semantic and syntactic information). Therefore, though skip-gram embeddings contain more information than a similarity graph, the information serves more as "noise" and less as useful information. Moreover, the robustness of affinity propagation is immune to the dimension of the embeddings, while others can be very sensitive to it. In our experiment, we find MeanShift is highly unstable if the dimension

\footnotetext{
${ }^{5}$ We set the preference to be -60 .
} 
is greater than 30. Hence affinity propagation, which takes similarities between pairs of data points as input, serves for our needs well.

\subsection{Discovering Coherent Semantic Facets of A Seed Set}

After obtaining multiple skip-gram clusters for each seed, we then need to determine the coherent semantic facets among all seeds and generate the coherent skip-gram clusters. Take two seed words "apple" with facets fruit and company, and "orange" with facets fruit and color as an example, their coherent semantic facet is fruit.

The key is to determine whether a facet of seed word $A$ matches any facet of word $B$. Suppose that $A$ has $r$ skip-gram clusters $S_{A}=\left\{S_{A}^{(1)}, \ldots, S_{A}^{(r)}\right\}$, where cluster $S_{A}^{(i)}$ contains a set of skip-grams relevant to the $i$-th facet of $A$. Similarly, $B$ has $t$ skip-gram clusters $S_{B}=\left\{S_{B}^{(1)}, \ldots, S_{B}^{(t)}\right\}$. If $A$ and $B$ share $k$ facets, and they have $k$ pairs of matching clusters $\left\{\left(S_{A}^{\left(i_{1}\right)}, S_{B}^{\left(j_{1}\right)}\right), \ldots,\left(S_{A}^{\left(i_{k}\right)}, S_{B}^{\left(j_{k}\right)}\right)\right\}$ accordingly. Therefore, these $k$ facets are jointly represented by these clusters:

$S_{A, B}=\left\{S_{A}^{\left(i_{1}\right)} \cup S_{B}^{\left(j_{1}\right)}, \ldots, S_{A}^{\left(i_{k}\right)} \bigcup S_{B}^{\left(j_{k}\right)}\right\}$.

We first measure the pairwise correlation of their skip-gram clusters (c.f. Sec 3.2 , and then make a matching decision on a pair of clusters (c.f. Sec 3.2).

Calculating correlation between two skip-gram clusters Suppose that facet $A_{1}$ (one facet of word $A$ ) corresponds to a skip-gram cluster $\mathbf{X}=\left[\mathbf{x}_{1} ; \ldots ; \mathbf{x}_{m}\right]$ with $m$ skip-gram vectors, where $\mathbf{x}_{i} \in \mathbb{R}^{d}$. Similarly, facet $B_{1}$ (one facet of word $B$ ) corresponds to a cluster $\mathbf{Y}=\left[\mathbf{y}_{1} ; \ldots ; \mathbf{y}_{n}\right]$ with $n$ skip-gram vectors, where $\mathbf{y}_{j} \in \mathbb{R}^{d}$. Two clusters $\mathbf{X}$ and $\mathbf{Y}$ are from different seed words, and we want to measure their correlation in order to decide whether they correspond to the same semantic facet.

To measure their correlation, we find the semantic sense which $\mathbf{X}$ and $\mathbf{Y}$ have in common. Inspired by the idea of compositional semantics [10]28, we set the sense vector to the linear combination of skip-gram vectors.

Suppose that the sense vector $\mathbf{u}$ from cluster $\mathbf{X}$ and the sense vector $\mathbf{v}$ from $\mathbf{Y}$ are the sense shared by the two clusters. Therefore, the common sense vectors should be highly correlated, i.e., we want to find $\mathbf{u}$ and $\mathbf{v}$ so that their correlation is maximized. We formulate the following optimization problem (1).

$$
\begin{array}{ll} 
& \max _{a, b} \frac{\mathbf{u}^{T} \mathbf{v}}{\|\mathbf{u}\| \cdot\|\mathbf{v}\|} \\
\text { s.t. } & \mathbf{u}=\mathbf{X a}, \\
& \mathbf{v}=\mathbf{Y} \mathbf{b},
\end{array}
$$

where $\mathbf{a} \in \mathbb{R}^{m}$ and $\mathbf{b} \in \mathbb{R}^{n}$ are coefficient vectors.

Solving the problem (1) by CCA 8 , we can find their common sense vectors $\mathbf{u}^{*}$ and $\mathbf{v}^{*}$. The semantic correlation $\operatorname{corr}(\mathbf{X}, \mathbf{Y})$ between cluster $\mathbf{X}$ and $\mathbf{Y}$ is defined as the correlation between these two sense vectors:

$$
\operatorname{corr}(\mathbf{X}, \mathbf{Y})=\mathbf{u}^{* T} \mathbf{v}^{*}
$$


Matching facets of all seeds After quantifying correlation for two skip-gram clusters, we cast it as a binary decision whether the cluster $\mathbf{X}$ of facet $A_{1}$ matches semantically with any facet of word $B$.

We note that it is not a good way to decide the matching clusters by setting a hard correlation threshold, since the the numerical correlation range is wordspecific. It is easy to see that if a facet of seed $A\left(e . g ., A_{1}\right)$ is of the same semantic class with a facet of seed $B\left(\right.$ e.g., $\left.B_{2}\right)$, then $\operatorname{corr}\left(A_{1}, B_{2}\right)$ is higher than the correlation between $A_{1}$ and any other facets of seed $B$. Otherwise, the correlation of $A_{1}$ and all facets of seed $B$ should be equally small.

Based on the intuition above, we define a relevance score below:

$$
\operatorname{rele}\left(A_{1}, B\right)=D_{K L}\left(\operatorname{Corr}\left(A_{1}, B\right), U\right)
$$

where $U$ is uniform distribution, $D_{K L}$ is KL-divergence [13], and $\operatorname{Corr}\left(A_{1}, B\right)=$ $\operatorname{softmax}\left(\left(\operatorname{corr}\left(A_{1}, B_{1}\right), \ldots, \operatorname{corr}\left(A_{1}, B_{m}\right)\right)\right)$,

We then make the matching decision based on the relevance score rele $\left(A_{1}, B\right)$. The threshold of the relevance score is set to 0.25 empirically. Once the matching decision is satisfied, we find the best matching facet $B^{*}$ in word $B$ and generate one coherent skip-gram cluster $A_{1} \bigcup B^{*}$. Finally, we do facet matching for all facets of word $A$ and obtain the resulting skip-gram clusters as coherent skipgram clusters.

Remarks: If there are more than two seed words, we first discover coherent skip-gram clusters of two seeds and then use their coherent skip-gram clusters to match with the third seed and so on.

\subsection{Entity Expansion}

The coherent skip-gram clusters of different facets are used to expand the seed set. Traditionally, researchers expands entites from a group of skip-gram clusters based on graph-based approaches [34, entity matching approaches [26, distributional hypothesis 22 and iterative approaches 35[9. As a distinction, we tackle the problem by a Masked Language Model (MLM), which leverages the discriminative power of the BERT model and contextual representations [5].

For each skip-gram denoted as $s g$, we compute the MLM probability $h_{c, s g}$ for each word candidate $c$ in the vocabulary by a pre-trained BERT model 6 Therefore, given a set of skip-grams, the weight $w_{c}$ of a word candidate $c$ is calculated as: $w_{c}=\sum_{s g^{\prime}} h_{c, s g^{\prime}}$. The final entity expansion process simply ranks all word candidates by their weights.

\section{Experiments}

Our model targets the corpus-based entity set expansion problem, and thus we evaluate its performance on a local corpus.

${ }^{6}$ We use the 'bert-base-uncased' pre-trained model from https://huggingface.co/ transformers/model_doc/bert.html\#bertformaskedlm. 
Table 1: Evaluation using MMAP ("recall"), PMAP ("precision") and BMAP ("F1 score").

\begin{tabular}{|c|c|c|c|c|c|c|c|c|c|c|}
\hline & & \multicolumn{3}{|c|}{ MMAP@l } & \multicolumn{3}{|c|}{ PMAP@l } & \multicolumn{3}{|c|}{ BMAP@l } \\
\hline & & $l=5$ & $l=\mathbf{1 0}$ & $l=\mathbf{2 0}$ & $l=5$ & $l=10$ & $l=\mathbf{2 0}$ & $l=5$ & $l=\mathbf{1 0}$ & $l=\mathbf{2 0}$ \\
\hline Single- & word2vec & 0.323 & 0.283 & 0.252 & 0.552 & 0.499 & 0.448 & 0.390 & 0.352 & 0.316 \\
\hline \multirow[t]{2}{*}{ Faceted } & SEISA & 0.345 & 0.301 & 0.268 & 0.550 & 0.503 & 0.455 & 0.408 & 0.368 & 0.331 \\
\hline & SetExpan & 0.373 & 0.337 & 0.304 & 0.605 & 0.563 & 0.512 & 0.448 & 0.413 & 0.374 \\
\hline Multi- & Sensegram & 0.312 & 0.301 & 0.275 & 0.479 & 0.443 & 0.398 & 0.359 & 0.343 & 0.314 \\
\hline \multirow[t]{2}{*}{ Faceted } & EgoSet & 0.446 & 0.390 & 0.325 & 0.306 & 0.261 & 0.206 & 0.335 & 0.292 & 0.236 \\
\hline & FUSE & 0.477 & 0.414 & 0.366 & 0.643 & 0.573 & 0.507 & 0.531 & 0.469 & 0.414 \\
\hline \multirow[t]{2}{*}{ Ablations } & FUSE-k $(k=\mathbf{2})$ & 0.420 & 0.364 & 0.326 & 0.607 & 0.540 & 0.494 & 0.478 & 0.422 & 0.383 \\
\hline & FUSE-k $(k=\mathbf{3})$ & 0.454 & 0.406 & 0.360 & 0.624 & 0.562 & 0.505 & 0.504 & 0.455 & 0.407 \\
\hline
\end{tabular}

Dataset: We evaluate our approach, FUSE, on the dataset in [22]. The dataset contains 56 million articles (1.2 billion words) retrieved from English Wikipedia 2014 Dump and 150 human-labeled multi-faceted queries.

\subsection{Evaluation Metric}

It is considerably complicated to properly evaluate multi-faceted set expansion task due to different number of facets between the generated result and the ground truth. Previous work 22] adopted the following Mean Mean Average Precision (MMAP) measure:

$$
M M A P @ l=\frac{1}{M_{q}} \sum_{m=1}^{M_{q}} A P_{l}\left(B_{q i^{*}}, G_{q m}\right),
$$

where $M_{q}$ is the number of facets for query $q$ in the ground truth; $G_{q m}$ is the ground truth set of $m$-th facet for $q ; B_{q i *}$ is the output facet that best matches $G_{q m}$, and $A P_{l}(c, r)$ represents the average precision of top $l$ entities in a ranked list $c$ given an unordered ground truth set $r$. This metric measures the coverage of ground truth sets by the generated sets.

However, it does not penalize additional noisy facets in generated sets and thus it is biased towards the model that generates more facets. For example, a model generating 30 facets with 3 relevant facets achieves higher MMAP than another model generating 3 facets with 2 relevant facets. One can "cheat" the performance by generating as many facets as possible.

To overcome the intrinsic limitation of MMAP, we, inspired by [7/3, propose a new metric, Best-Matching Average Precision (BMAP) to capture both the purity of generated facets and their coverage of ground truth facets. Our metric is defined as follows:

$$
B M A P @ l=H M e a n(M M A P @ l, P M A P @ l),
$$




$$
P M A P @ l=\frac{1}{F_{q}} \sum_{f=1}^{F_{q}} A P_{l}\left(B_{q f} ; G_{q i^{*}}\right),
$$

where $F_{q}$ is the number of facets in generated output; $B_{q f}$ is the $f$-th output ranked list for query $q ; G_{q i}$ is the ground truth facet that best matches $B_{q f}$. Here $H$ Mean $(a, b)=\frac{2 a b}{a+b}$ is the harmonic mean of $a$ and $b$.

Our proposed BMAP metric not only evaluates how well generated facets match the ground truth by $M M A P @ l$ but also penalizes low-quality facets by $P M A P @ l$. Intuitively, $M M A P @ l$ measures "recall" to capture how many ground truth results has been discovered, while PMAP@l measures "precision" to capture the fraction of good facets in the generated output. Accordingly, $B M A P @ l$ measures "F1 score" to leverage "precision" and "recall". Results are reported by averaging all queries for each dataset.

\subsection{Baselines}

The following approaches are evaluated:

- word2ved7 [17: We use the "skip-gram" model in word2vec to learn the embedding vector for each entity, and then return $k$ nearest neighbors of the seed words.

- SEISA 9]: An entity set expansion algorithm based on iterative similarity aggregation. It uses the occurrence of entities in web list and query log as entity features. In our experiments, we replace the web list and query log with skip-gram features.

- SetExpan 26: A corpus-based set expansion that selects quality context features for entity-entity similarity calculation and expand the entity sets using rank ensemble.

- EgoSet 22]: The only existing work for multi-faceted set expansion. It expands word entities from skip-gram features, and then clusters the expanded entities into multiple sets by the Louvain community detection algorithm.

- Sensegram ${ }^{9}$ 19]: We learn different embeddings for each word's different senses and return $k$ nearest neighbors for each embedding.

- FUSE-k: A variant of FUSE which replaces Affinity Propagation with $k$ means clustering algorithm for skip-gram clustering.

\section{$4.3 \quad$ Results}

We compare the performance of FUSE against all baselines using MMAP ("recall"), PMAP ("precision") and BMAP ("F1 score"). As shown in Table 1. FUSE achieves the highest scores in most cases and outperforms all other baselines in BMAP and MMAP.

\footnotetext{
7 https://code.google.com/p/word2vec

8 https://github.com/mickeystroller/SetExpan

9 https://github.com/uhh-lt/sensegram
} 
It is worth mentioning that EgoSet achieves decent results in MMAP. However, it generates too many noisy facets, which deteriorate PMAP and the overall performance BMAP. We will further discuss this phenomenon in Sec. 4.4.

It is also interesting to note that single-faceted baselines (i.e., SetExpan, SEISA) have much stronger PMAP performance than multi-faceted baselines. This is because by generating a single cluster of the most confident expansion results, they usually match with one ground truth cluster very well and thus achieve high PMAP ("precision") value.

In the ablation analysis, it is worth noting that FUSE, even without predetermined number of clusters, performs better than FUSE-k. We experiment the number of clusters $k$ to be 2 and 3 , which are the mode and the mean of the number of clusters of the ground truth respectively. We think the poor performance is because forcing skip-grams into a fixed number of clusters will induce clustering noise. Furthermore, the noise will propagate and be enlarged in the skip-gram cluster fusion step and the entity expansion step, and therefore leads to bad performance.

\subsection{Number of Facets Identified}

We explore the number of facets identified by different multi-faceted set expansion methods. Specifically, we adopt $l_{1}$ and $l_{2}$ distances.

$$
\begin{array}{r}
l_{1} \text { distance }=\sum_{q \in Q}\left|\mathrm{GT}_{q}-\mathrm{Gen}_{q}\right| \\
l_{2} \text { distance }=\sqrt{\sum_{q \in Q}\left(\mathrm{GT}_{q}-\mathrm{Gen}_{q}\right)^{2}}
\end{array}
$$

Here $Q$ is all queries, $\mathrm{GT}_{q}$ and $\mathrm{Gen}_{q}$ are the number of facets that ground truth has and the number of facets that the corresponding model identifies for query $q$, respectively.

The distance measurement are summarized in Table 2 FUSE generates closer number of facets to the ground truth, compared to EgoSet, demonstrating about $65 \%$ reduction of the $l_{1}$ distances and $45 \%$ reduction of the $l_{2}$ distances.

Table 2: Distance between the number of facets identified and the number of facets the ground truth has.

\begin{tabular}{lcc}
\hline & $l_{1}$ distance & $l_{2}$ distance \\
\hline EgoSet & 783 & 78.02 \\
\hline FUSE & $\mathbf{2 7 7}$ & $\mathbf{4 3 . 0 5}$ \\
\hline
\end{tabular}


Table 3: Case study on comparison between FUSE and EgoSet.

\begin{tabular}{|c|c|c|}
\hline Query & $\begin{array}{l}\text { FUSE Identified Facets and Their Associated } \\
\text { Top Skip-grams }\end{array}$ & EgoSet Identified Facets and Their Associated Top Skip-grams \\
\hline \multirow{7}{*}{ berkeley\} } & \multirow{4}{*}{$\begin{array}{l}\text { Facet } 1 \text { (Top universities in the US): } \\
\text { columbia_university, harvard, new_york, } \\
\text { yale_university, princeton, harvard_university, } \\
\text { ucla, stanford_university, columbia, } \\
\text { boston_college, nyu, cornell_university, } \\
\text { indiana_university, johns_hopkins_university, } \\
\text { georgetown, georgetown_university, ,.. } \\
\text { Top Skip-grams: ('at_, yale'), ('graduate } \\
\text { school at_,'), ('former__ professor'), (', _, } \\
\text { harvard university'), (', _ campus .') }\end{array}$} & $\begin{array}{l}\text { Facet 1: los_angeles, san_diego, san_francisco, santa_barbara, santa_cruz, riverside, } \\
\text { sacramento, ... } \\
\text { Top Skip-grams: ('in _, ca.'), ('to _ , california .') }\end{array}$ \\
\hline & & $\begin{array}{l}\text { Facet 2: stanford, harvard_university, yale_university, columbia, cornell_university, mit, } \\
\text { ucsd, ... } \\
\text { Top Skip-grams: ('the__campus.'), ('at__ 's school of') }\end{array}$ \\
\hline & & $\begin{array}{l}\text { Facet 3: chicago, san_leandro, huntington_beach, los_angeles_county, oxnard, van_nuys, } \\
\ldots \\
\text { Top Skip-grams: ('in__, los'), ('in _, los angeles') } \\
\end{array}$ \\
\hline & & Facet 4: culver_city, santa_ana, costa_mesa, alameda, redondo_beach, san_rafael, fullerton \\
\hline & \multirow{3}{*}{ 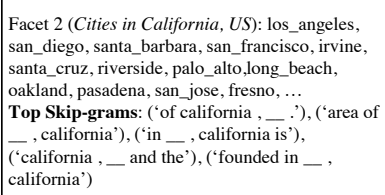 } & Top Skip-grams: ('city of _, california'), ('in _, california where') \\
\hline & & $\begin{array}{l}\text { Facet 5: california_state_university, marine_corps_recruit_depot, howard_university, } \\
\text { california_polytechnic_state_university, uc_santa_barbara, scripps, ... } \\
\text { Top Skip-grams: ('at__, san'), ('of california ___,') } \\
\end{array}$ \\
\hline & & $\begin{array}{l}\text { Facet 6: ojai, yuba_city, whittier_college, pasadena_city_college, mather_air_force_base, } \\
\text { moffett_field, beale_afb, march_field, beale_air_force_base, fort_ord, ... } \\
\text { Top Skip-grams: ('at__, california.'), ('at__, california in') }\end{array}$ \\
\hline
\end{tabular}

To further explore identified facets between FUSE and EgoSet, we present one case study of query \{ "berkeley" $\}$ in Table 3. FUSE generates two facets and each facet has its distinctive semantic meaning (one for Top universities in the US and one for Cities in California, US), while EgoSet generates too many scattered facets, with less distinctiveness between facets. One of the reasons is that EgoSet performs clustering on expanded entities while FUSE performs clustering on skip-grams. Skip-grams, consisting of multiple words, are usually of more clear semantics compared to entities themselves (e.g., the entity "columbia" can be either Universities, Cities or Rivers, while the skip-gram "graduate school at _-" is more clear to be Universities.). Therefore, clustering on skip-grams is an easier process and results in better performance.

\subsection{Case Studies: Multi-Faceted Setting}

Table 4 shows intermediate results of FUSE by listing top skip-grams of each semantic facet. It is worth noting that even the ground truth may not present a full coverage of semantic facets of given seeds. For example, as shown in Case 4, the ground truth only includes semantic facet Animals. Our system also finds another meaningful semantic facet Tributaries ${ }^{10}$ The query $\{$ "Chongqing" $\}$ shown in Case 5 is another example, where the ground truth again fails to capture the semantic facet of War-related Major Cities ${ }^{11}$

${ }^{10}$ Beaver River, Elk River and Bear River are tributaries of Pennsylvania, Mississippi River and the Great Salt Lake, respectively.

${ }^{11}$ Chongqing was the second capital of Chinese nationalist party during the war. 
Table 4: Case studies on top skip-grams for each semantic facet. The concept name of each facet is in bold.

\begin{tabular}{|c|c|c|c|}
\hline ID & Query & FUSE Identified Facets: Associated Top Skip-grams & Ground Truth Facets: Example Entities \\
\hline 1 & $\begin{array}{l}\text { \{hydrogen, } \\
\text { uranium\} }\end{array}$ & $\begin{array}{l}\text { Chemical Elements: (helium, }, \text {,), (of _ and nitrogen.) } \\
\text { Energy Sources: (for _ energy), (in__energy), (the _ fuel cell) }\end{array}$ & $\begin{array}{l}\text { Chemical Elements: Helium, Carbon, Nitrogen, Oxygen } \\
\text { Energy Sources: Solar, Coal, Oil, Natural Gas }\end{array}$ \\
\hline 2 & $\begin{array}{l}\text { \{apollo, } \\
\text { artemis, } \\
\text { poseidon\} }\end{array}$ & $\begin{array}{l}\text { Major gods: (the god _and), (zeus, _, }),(\text {, athena , _, }) \\
\text { NASA missions: (nasa 's _, ) }\end{array}$ & $\begin{array}{l}\text { Major Greek gods: Aphrodite, Ares, Athena, Zeus } \\
\text { NASA missions: Juno, Voyager, InSight, NuSTAR }\end{array}$ \\
\hline 3 & $\{$ Beijing $\}$ & 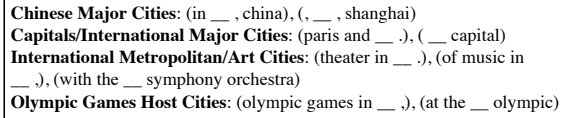 & $\begin{array}{l}\text { Chinese Major Cities: Beijing, Shanghai, Wuhan, Harbin } \\
\text { Province-level divisions of China: Beijing, Jiangsu, Zhejiang } \\
\text { Capital cities in the world: Paris, Tokyo, Jakarta, Berlin } \\
\text { Olympic Games Host Cities: Paris, Tokyo, Munich, Calcary }\end{array}$ \\
\hline 4 & $\begin{array}{c}\left\{\begin{array}{c}\text { beaver, elk, } \\
\text { bear }\}\end{array}\right. \\
\text {. }\end{array}$ & $\begin{array}{l}\text { Animals: (tailed deer , _ }, \text { ), (wolf, }, \text { and) } \\
\text { Tributaries: (in _ river,), (along the _ river.) }\end{array}$ & Animals: alligator, bear, deer, pig \\
\hline 5 & $\{$ Chongqing\} & $\begin{array}{l}\text { Chinese Major Cities: (of _, china.), (based in _, china.) } \\
\text { War related Major Cities: (_ broadcasting), (congress of _ .), (party in } \\
\text { _ led by) }\end{array}$ & $\begin{array}{l}\text { Chinese Major Cities: Beijing, Shanghai, Wuhan, Harbin } \\
\text { Province-level divisions of China: Beijing, Jiangsu, Zhejiang, } \\
\text { Guangdong }\end{array}$ \\
\hline 6 & $\{f o x\}$ & 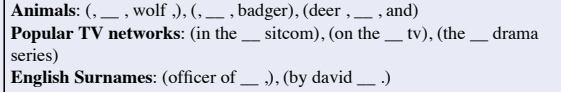 & $\begin{array}{l}\text { Animals: alligator, bear, deer, pig } \\
\text { Popular TV networks: NBC, CBS, CNN } \\
\text { Common English surnames: Smith, Jones, Williams } \\
\text { Snake species: Blind snake, Sea snake, Python }\end{array}$ \\
\hline
\end{tabular}

\section{Single-Faceted Set Expansion}

In previous sections, we have demonstrated that FUSE has favorable performance against state-of-the-art systems in expanding multiple semantic facets of a seed set. Yet, it's more common in real-life cases that a seed set has one single semantic facet (especially when more seeds are provided). In this subsection, we inspect the performance of FUSE on single-faceted cases.

In the singled-faceted set expansion task, there is exactly one semantic facet from a seed set. However, if one or more words in the seed set are ambiguous, such ambiguity will introduce entities related to noisy facets, and thus hurt the quality of the expanded set. For example, the seed set \{ "apple", "amazon" $\}$ has only one semantic facet corresponding to Technology Companies, however, the seed "apple" is an ambiguous word and has a noisy facet, i.e., Fruits. Most existing systems 3134 35 18 25 9 33 2 26 15 22 first extract all contextual features (e.g., skip-grams) from the entire seed set and then rank the keyword candidates based on the contextual features. To the best of our knowledge, none of them denoises contextual features from the noisy facet (i.e., Fruits). Therefore, they are likely to generate entities related to the facet of Fruits (e.g., "pear", "banana"), despite the fact that the seed set \{ "apple", "amazon" $\}$ has only one semantic facet.

In contrast, FUSE is robust to such lexical ambiguity, since we discover the shared coherent semantic facet across all seeds and expand entities by relevant contextual features only. From this example, one can clearly see that even for single-faceted set expansion, it is also critical to resolve the lexical ambiguity and identify the common facet among seeds. 
To gain deeper insights in the single-faceted setting, we present a case study on the seed set $\{$ "apple", "amazon" $\}$ in Table 5. We highlight those noisy entities resulting from seed ambiguity (i.e., the Fruits sense) in bold, bright red. As expected, EgoSet has 2 facets that contain entites from the Fruits sense and SetExpan suffers from the noisy facet issue too. As it has shown, FUSE performs favorably against previous approaches in single-faceted set expansion too, in that it is robust to semantic ambiguity by extracting the coherent semantic facet shared by all seed words.

Table 5: Case studies on single-faceted set expansion: $\{$ "apple", "amazon" $\}$.

\begin{tabular}{|c|c|}
\hline Approach & Expanded Entities of query \{“apple”, “amazon”\} \\
\hline Ground Truth & Technology Companies: samsung electronics, foxconn, hp, ibm, amazon, microsoft, sony, panasonic, dell, intel, toshiba, ... \\
\hline FUSE & $\begin{array}{l}\text { google, microsoft, sony, ibm, intel, general_electric, motorola, ebay, hewlett_packard, coca_cola, syntel, idw_publishing, } \\
\text { corus_entertainment, black_isle, independent_record_label, grunewald, facebook, ... }\end{array}$ \\
\hline SetExpan & united_states, pear, blackberry, american, strawberry, palm, blueberry, green, cherry, google, company, banana, gnu/linux, ... \\
\hline EgoSet & $\begin{array}{l}\text { Facet 1: microsoft, google, ibm, compaq, oracle, avaya, ebay, motorola, netflix, aol, virtualization, novell, general_mills, digital, ... } \\
\text { Facet 2: amazon_mp3, napster, adobe_photoshop, imovie, windows_media_player, itunes, iphone, mozilla_application_suite, ... } \\
\text { Facet } 3: \text { cp___m, microsoft_windows, apple_inc, as/400, vms, openstep, oem, vax, qnx, android, solaris, unix, smartphone,... } \\
\text { Facet 4: amiga, autodesk, gp, microcontroller, laptop, smithfield_foods, liberty_global, medtronic, digital_research, dr_pepper, ... } \\
\text { Facet 5: fasta, ntfs, wav, gemstone, nautilu } \\
\text { Facet 6: avocado, fruit, palm, blackberry, tapioca, manure, cochineal, musk, chocolate, horse_meat, symantec, lime_juice, ... } \\
\text { Facet 7: asia, milk, north_america, blueberry, pomegranate, grapefruit, flash, mobile, betty_crocker, xcode, cream_soda, pxe, ... } \\
\text { Facet } 8 \text { : fish, french_fry, consumer_electronic, debian, burger_king, poultry, coconut_oil, kool_aid, lobster, starch, byte, beef, ... }\end{array}$ \\
\hline
\end{tabular}

\section{Related Work}

Early work on entity set expansion, including Google Sets [31, SEAL [34, and Lyretrail 2 submits a query consisting of seed entities to a general-domain search engine (e.g., Google) and then mines the returned, top-ranked web pages. These approaches depend on the external search engine and require costly online data extraction.

Later studies shift to the corpus-based set expansion setting, where sets are expanded within a given domain-specific corpus. For example, [18] compute the semantic similarity between two entities based on their local contexts and treat the nearest neighbors around the seed entities as the expanded set. 9] further extend this idea by proposing an iterative similarity aggregation function to calculate entity-entity similarity using query logs and web lists besides free text. More recently, 26 27] propose to compute semantic similarity using only selected high-quality context features, and 1415] develop SetExpander system to leverage multi-context term embedding for entity set expansion. All the above attempts, however, assume the input seed entities belong to one unique, clear semantic class, and thus largely suffer from the multi-faceted nature of these seeds - they could represent multiple semantic meanings.

To resolve the ambiguity of seeds, 33. propose to utilize the target semantic facet name and then retrieve its most relevant web tables. However, it requires 
the exact name of the target semantic facet and outputs the one semantic facet of entities only. This does not accomplish multi-faceted set expansion in nature.

The only attempt towards multi-faceted set expansion is EgoSet [22, to the best of our knowledge. EgoSet first extracts quality skip-gram features to construct an entity-level ego-network, and then perform the Louvain community detection algorithm on the ego-network to extract entities for different facets. Finally, it combines them with external knowledge (i.e., user-generated ontologies) to generate final output. Although FUSE may appear to be similar to EgoSet at first glance, we highlight key differences and significance below:

- Key challenges of multi-faceted set expansion: We identify the key challenge of multi-faceted set expansion to be discovery of shared semantic facets from a seed set. While in EgoSet, noisy facets that are relevant only to partial seeds are also generated. As a result, it fails to solve multi-seed singlefaceted cases (e.g., \{ "apple", "amazon" $\})$ and multi-seed multi-faceted cases (e.g., Fig. 1).

- External knowledge: EgoSet requires user-created ontology (obtained from Wikipedia) as external knowledge. While these semi-structured web tables and ontologies are helpful for disambiguation, they are not always available for domain-specific corpus. FUSE relies on free text only and thus, can be applied in a more general setting.

- Clustering over skip-grams: EgoSet adopts clustering (community detection) over expanded entities, while FUSE adopts clustering over skip-grams. Clustering over entities usually leads to mediocre results in non-parametric settings, since any expanded entity can be ambiguous. However, skip-grams, consisting of multiple words, are usually of more clear semantics and much easier to be clustered compared to entities themselves (demonstrated in Sec. 4.4 . In additional, EgoSet adopts hard clustering on entities, which ignores the nature that the same entity may fall into different facets (e.g., "Paris" should appear in both sets of National Capitals and Olympic Games Host Cities in Fig. 1), while the design of FUSE naturally allows the same entities to appear in multiple facets.

In a more general way, our work is also related to word sense disambiguation [30|20|21|11|19]. The major difference is that our work aims to find the coherent semantic facets of all seed words and achieve entity expansion from the coherent skip-gram clusters.

\section{Conclusion}

We identify the key challenge of the problem - multi-faceted set expansion and have proposed a novel and effective approach, FUSE, to address it. By extracting and clustering skip-grams for each seed, identifying coherent semantic facets of all seeds, and expanding entity sets for each semantic facet, FUSE is capable of identifying coherent semantic facets, generating quality entity set for each facet, and therefore outperforms previous state-of-the-art approaches significantly. 
The proposed framework FUSE is general in that it achieves quality set expansion in both multi-faceted and single-faceted settings. In particular, it, for the first time, solves the case where different seeds have different multi-facetedness. For future work, we plan to explore other skip-gram clustering approaches and coherent semantic facet discovery algorithms.

Acknowledgments Research was sponsored in part by US DARPA KAIROS Program No. FA8750-19-2-1004 and SocialSim Program No. W911NF-17-C0099, National Science Foundation IIS 16-18481, IIS 17-04532, and IIS-17-41317, and DTRA HDTRA11810026.

\section{References}

1. Cao, H., Jiang, D., Pei, J., He, Q., Liao, Z., Chen, E., Li, H.: Context-aware query suggestion by mining click-through and session data. In: KDD (2008)

2. Chen, Z., Cafarella, M., Jagadish, H.: Long-tail vocabulary dictionary extraction from the web. In: WSDM (2016)

3. Chinchor, N.: Muc-4 evaluation metrics. In: Proceedings of the 4th conference on Message understanding. Association for Computational Linguistics (1992)

4. Comaniciu, D., Meer, P.: Mean shift: A robust approach toward feature space analysis. IEEE Transactions on pattern analysis and machine intelligence 24(5), 603-619 (2002)

5. Devlin, J., Chang, M.W., Lee, K., Toutanova, K.: Bert: Pre-training of deep bidirectional transformers for language understanding. In: NAACL (2019)

6. Frey, B.J., Dueck, D.: Clustering by passing messages between data points. science 315(5814), 972-976 (2007)

7. Goldberg, M.K., Hayvanovych, M., Magdon-Ismail, M.: Measuring similarity between sets of overlapping clusters. In: Social Computing (SocialCom), 2010 IEEE Second International Conference on. pp. 303-308. IEEE (2010)

8. Hardoon, D.R., Szedmak, S., Shawe-Taylor, J.: Canonical correlation analysis: An overview with application to learning methods. Neural computation 16(12), 2639$2664(2004)$

9. He, Y., Xin, D.: Seisa: set expansion by iterative similarity aggregation. In: WWW (2011)

10. Hermann, K.M., Blunsom, P.: Multilingual models for compositional distributed semantics. arXiv preprint arXiv:1404.4641 (2014)

11. Iacobacci, I., Pilehvar, M.T., Navigli, R.: Embeddings for word sense disambiguation: An evaluation study. In: ACL (2016)

12. Jain, A., Pennacchiotti, M.: Open entity extraction from web search query logs. In: COLING (2010)

13. Kullback, S., Leibler, R.A.: On information and sufficiency. The annals of mathematical statistics 22(1), 79-86 (1951)

14. Mamou, J., Pereg, O., Wasserblat, M., Dagan, I., Goldberg, Y., Eirew, A., Green, Y., Guskin, S., Izsak, P., Korat, D.: Setexpander: End-to-end term set expansion based on multi-context term embeddings. In: COLING (2018)

15. Mamou, J., Pereg, O., Wasserblat, M., Eirew, A., Green, Y., Guskin, S., Izsak, P., Korat, D.: Term set expansion based nlp architect by intel ai lab. In: EMNLP (2018) 
16. Mikolov, T., Sutskever, I., Chen, K., Corrado, G.S., Dean, J.: Distributed representations of words and phrases and their compositionality. In: NIPS (2013)

17. Mikolov, T., Sutskever, I., Chen, K., Corrado, G.S., Dean, J.: Distributed representations of words and phrases and their compositionality. In: NIPS (2013)

18. Pantel, P., Crestan, E., Borkovsky, A., Popescu, A.M., Vyas, V.: Web-scale distributional similarity and entity set expansion. In: EMNLP (2009)

19. Pelevina, M., Arefyev, N., Biemann, C., Panchenko, A.: Making sense of word embeddings. In: Rep4NLP@ACL (2016)

20. Raganato, A., Bovi, C.D., Navigli, R.: Neural sequence learning models for word sense disambiguation. In: EMNLP (2017)

21. Raganato, A., Camacho-Collados, J., Navigli, R.: Word sense disambiguation: A unified evaluation framework and empirical comparison. In: EACL (2017)

22. Rong, X., Chen, Z., Mei, Q., Adar, E.: Egoset: Exploiting word ego-networks and user-generated ontology for multifaceted set expansion. In: WSDM (2016)

23. Sarker, A., Gonzalez, G.: Portable automatic text classification for adverse drug reaction detection via multi-corpus training. Journal of biomedical informatics 53 , 196-207 (2015)

24. Sarmento, L., Jijkoun, V., de Rijke, M., Oliveira, E.C.: "more like these": growing entity classes from seeds. In: CIKM (2007)

25. Sarmento, L., Jijkuon, V., De Rijke, M., Oliveira, E.: More like these: growing entity classes from seeds. In: CIKM (2007)

26. Shen, J., Wu, Z., Lei, D., Shang, J., Ren, X., Han, J.: Setexpan: Corpus-based set expansion via context feature selection and rank ensemble. In: ECML/PKDD (2017)

27. Shen, J., Wu, Z., Lei, D., Zhang, C., Ren, X., Vanni, M.T., Sadler, B.M., Han, J.: Hiexpan: Task-guided taxonomy construction by hierarchical tree expansion. In: KDD (2018)

28. Socher, R., Karpathy, A., Le, Q.V., Manning, C.D., Ng, A.Y.: Grounded compositional semantics for finding and describing images with sentences. Transactions of the Association of Computational Linguistics 2(1), 207-218 (2014)

29. Steinbach, M., Ertöz, L., Kumar, V.: The challenges of clustering high dimensional data. In: New directions in statistical physics, pp. 273-309. Springer (2004)

30. Taghipour, K., Ng, H.T.: Semi-supervised word sense disambiguation using word embeddings in general and specific domains. In: NAACL-HLT (2015)

31. Tong, S., Dean, J.: System and methods for automatically creating lists (2008), uS Patent $7,350,187$

32. Velardi, P., Faralli, S., Navigli, R.: Ontolearn reloaded: A graph-based algorithm for taxonomy induction. Computational Linguistics 39(3), 665-707 (2013)

33. Wang, C., Chakrabarti, K., He, Y., Ganjam, K., Chen, Z., Bernstein, P.A.: Concept expansion using web tables. In: WWW (2015)

34. Wang, R.C., Cohen, W.W.: Language-independent set expansion of named entities using the web. In: ICDM (2007)

35. Wang, R.C., Cohen, W.W.: Iterative set expansion of named entities using the web. In: ICDM (2008)

36. Weikum, G., Theobald, M.: From information to knowledge: harvesting entities and relationships from web sources. In: PODS. pp. 65-76. ACM (2010)

37. Zhao, J., Liu, K., Zhou, G., Qi, Z., Liu, Y., Han, X.: Knowledge extraction from wikis/bbs/blogs/news web sites. (2014)

38. Zhu, W., Zhang, C., Yao, S., Gao, X., Han, J.: A spherical hidden markov model for semantics-rich human mobility modeling. In: AAAI (2018) 